\title{
«Ein Haufen Steine hat noch nie ein Haus ergeben»
}

Ariane Treu, Maurice Hurni, Georges Gabris,

Lito Panayotopoulos, Nick Miller, Alain Porchet

Comité du Groupement des psychiatres-psychothérapeutes membres de la Société Vaudoise de Médecine GPPV

Übersetzung:

Dr. med. Stefan Zlot

\footnotetext{
Zur besseren Lesbarkeit wird nur die männliche Form verwendet, gemeint sind beide Geschlechter.
}

Korrespondenz:

Dr. Ariane Treu

Spécialiste en psychiatrie et psychothérapie FMH Spécialiste en psychiatrie et psychothérapie d'enfants et d'adolescents FMH

Vice-présidente du GPPV

Rue Beau-Séjour 7

CH-1003 Lausanne

a.treu@bluewin.ch
Der in der Überschrift zitierte Satz des Mathematikers Henri Poincaré kommt einem bei der Lektüre des Fragebogens in den Sinn, den die Psychiater* zur Deklaration ihrer Qualitätsvorhaben im Rahmen des Projektes «Qualitätsmonitoring» der FMH erhalten haben.

Sinn dieser ersten Etappe ist es, die Qualitätsaktivitäten der Psychiater zu erfassen, um sie statistisch sichtbar zu machen. Die erhobenen Daten sollen hinsichtlich Kosten und Nutzen analysiert und die Ärzte anschliessend aufgrund ihrer Qualitätsaktivitäten evaluiert werden. Es ist vorgesehen, die Resultate zu publizieren, damit die Ärzte miteinander verglichen werden können und die Konkurrenz in Bezug auf Qualität und Preis zu spielen beginnt [1].

Der Doppelsinn des Projektes wird zunehmend offensichtlich.

$\mathrm{Zu}$ Beginn handelte es sich gemäss FMH darum, «die Arbeit der Ärzteschaft sichtbarer zu machen, um die Qualität der Behandlungen zu garantieren» [1] Unmerklich und schleichend erfolgt im Namen der Transparenz eine Transformation dieses Anspruchs hin zur Errichtung eines statistischen Verwaltungs- und Kontrolldispositives. Gestützt auf formelle Produktivitätskriterien, werden dabei unternehmerische Normen und die Idee der marktwirtschaftlichen Konkurrenz in unsere medizinische Praxis eingeführt, die bis anhin vor solchen Tendenzen bewahrt werden konnte.

In der Tat sind die Bedingungen zur Ausübung der psychiatrischen Medizin aktuell auf vielfältige Weise geregelt und kontrolliert: Nach Erwerb des ärztlichen Diploms ist eine Spezialisierung von mindestens 5 Jahren nötig, um den FMH-Titel Psychiatrie und Psychotherapie zu erlangen. Danach werden pro 3 Jahre 240 Stunden obligatorischer Fortbildung verlangt, die mit einem Fortbildungsdiplom für den jeweiligen Zeitraum attestiert wird. Einen bedeutenden Teil davon nehmen die regelmässigen Supervisionen und Intervisionen ein, die als direkte Qualitätskontrolle betrachtet werden können.

Der Psychiater muss den Vertrauensärzten der Krankenkassen regelmässig Bericht über seine Arbeit erstatten, um die Angemessenheit seiner therapeutischen Indikationen und der Kosten zu rechtfertigen. Er ist der Wirtschaftlichkeitskontrolle seiner Therapien durch santésuisse (ANOVA Index) unterworfen. Unzufriedene Patienten können sich an die Kommmission für Deontologie der kantonalen Ärztegesellschaft oder an die Justiz wenden. Diese kantonalen Ärztegesellschaften und ihre Spezialarztgruppierungen sind eben- falls Elemente der Ethik- und Qualitätskontrolle der Behandlungen. Dies sollte gesagt werden, um all die existierenden Massnahmen sichtbar zu machen, nicht um sie zu disqualifizieren. Nehmen wir einen Chauffeur, der seinen Fahrausweis gemacht hat, der regelmässig Kurse zur Perfektionierung seiner fahrerischen Fähigkeiten absolviert, die wiederum alle 3 Jahre durch ein Diplom validiert werden, der bei der Ausübung seines Berufs nie Probleme gehabt hat, der von seinen Berufskollegen geschätzt wird, dem sich die Passagiere freiwillig anvertrauen: Werden wir von einem solchen Chauffeur verlangen, dass er transparent belegt, warum er gut fährt? Die Absurdität dieser Vorgabe ist offensichtlich.

Doch die Lektüre des «Qualitätsfragebogens» bringt zusätzliche Überraschungen. Neben einer weiteren Erfassung der Fortbildungsaktivitäten (Rubriken A und C) - völlig unnötig, da bereits existierend - zeugen die übrigen Punkte von einer bürokratisch-verwalterischen Wahrnehmung der klinischen Aktivität: Verifizierung der Sicherheit und der Handhabung der Notfälle, der Triagekonzepte, Formalisierung der Beziehungsaspekte durch Meinungsumfragen, Kontrollen, Organisations- und Verwaltungssystem, Zertifizierung der Praxis. Die gewählten Kriterien stellen genau die Werte in Frage, auf denen diese Evaluation beruhen sollte.

\section{Organisation, Kontrolle und Verwaltung statt} Relevanz, Korrektheit und Verständlichkeit

Entsprechend den Empfehlungen der Schweizerischen Akademie der Medizinischen Wissenschaften sollten die Erhebung, die Analyse sowie die Publikation von Daten, welche die Qualität medizinischer Behandlungen betreffen, drei Kriterien erfüllen: Relevanz, Korrektheit und Verständlichkeit. Messen bedeutet eine vorgängige Auswahl relevanter Kriterien, welche die Qualität charakterisieren. Transparenz sollte bestehen bei der Wahl dieser Kriterien, und der Verantwortliche für diese Auswahl sollte bekannt sein [2].

Die Parameter jedoch, die für diesen Fragebogen gewählt wurden, betreffen vorrangig Werte, die sich auf betriebswirtschaftliche Prinzipien, Organisation, Kontrolle und Verwaltung abstützen. Einige Kriterien sind nicht nur absurd, sondern kontraproduktiv. Sie gefährden die therapeutische Beziehung - das hauptsächliche Instrument des ärztlichen Psychotherapeuten - indem sie diese vergegenständlichen. Wenn ein Fragebogen über die Zufriedenheit mit einer Leistung in der Hotellerie sinnvoll sein kann, so ist die Vorstel- 
lung, dass auf diese Weise die Qualität der psychotherapeutischen Arbeit charakterisiert werden kann, ein Zeichen für erschreckende Unkenntnis zentraler Begriffe wie Verführung, Ambivalenz, Spaltung oder Destruktivität. Die ärztliche Handlung wird zu einer Ware, der Psychiater zu einem netten und gut organisierten Coach.

Nachdem die Bedeutung der Komplexität in der klinischen Medizin genauso anerkannt ist wie die Erkenntnis, dass der Patient sich nicht auf die Krankheit reduzieren lässt und dass die Krankheit selbst sowie deren Behandlung komplexe Prozesse sind - von welcher Qualität sprechen wir da?

Die in den Qualitätsvorgaben geforderte Transparenz hat nichts mit der Wahrheit zu tun, sie bezeichnet die Inkonsistenz und die Leere, die Gestalt einer formellen, ihres klinischen Inhalts entleerten Medizin.

Unter dem Vorwand der Transparenz, indem die Evaluation unserer Arbeit auf eine oberflächliche und konformistische statistische Verwaltung reduziert wird, werden wir bezifferbare und standardisierte Leistungen erbringen können (Transparenz, die auf sich selbst anzuwenden sogar die Versicherer verweigern, aus anderen Gründen). Gleichzeitig werden wir jedoch genau die zentralen Bedingungen der Behandlung zerstören: die privilegierte Beziehung zwischen dem Patienten und seinem Arzt, die Respektierung der Intimsphäre des Patienten durch die Garantie des Arztgeheimnisses, die Priorität der Klinik gegenüber der administrativen Verwaltung, die individualisierte Behandlung, die Berücksichtigung der Subjektivität, den Geist der Verantwortung und das persönliche Engagement.

Wenn wir gegen diesen Fragebogen Stellung beziehen, dann nicht nur deshalb weil er zur Evaluation der Qualität unserer Arbeit eine Sammlung absurder Daten erhebt, sondern vor allem deshalb, weil die Annahme eines solchen Dispositivs zur Evaluation der Qualität wiederum die Praxis beeinflusst, indem eine neue Norm konstituiert wird. «Es genügt, dass sich der

Kommentar der SGPP* und der SGKJPP** zum Artikel von Ariane Treu et al.

In den beiden zitierten Artikeln der SÄZ wird, wie bereits von Kollegin Treu ausgeführt, auf die problematischen und eventuell kontraproduktiven Seiten von Qualitätsmessungen und -definitionen hingewiesen. Sowohl die Qualitätsbeauftragten unserer beiden Fachgesellschaften wie auch FMHVerantwortliche sind sich dieser Klippen wohl bewusst. Durch das KVG sind wir seit gut 15 Jahren verpflichtet, Qualität nachzuweisen. Unsere Bemühungen entspringen deshalb weder vorauseilendem Gehorsam noch Übereifer. Wenn wir nichts tun, bleibt es bei unbefriedigenden WZW-Kriterien oder anderen von «aussen» aufgezwungenen Messgrössen.

Das Projekt Q-Monitoring ist keine Qualitätsmessung, sondern eine Erfassung der Qualitätsaktivitä-
Aktivitätsindikator in eine Leistungsnorm umwandelt, damit sich die Ärzte schliesslich in ihrem eigenen Interesse dieser Norm angleichen: So gelangt man von der Evaluation zur freiwilligen Knechtschaft» [3]. Somit handelt es sich um einen Paradigmenwechsel in der psychiatrischen Behandlung, der seinen Namen verschweigt.

Dagegen leitet sich der Wert in diesem System von der sozialen Sichtbarkeit ab. Die Katastrophe dieses Prozesses sieht man gut im Bereich der Forschung, wenn die Forscher nicht mehr aufgrund des Inhaltes ihrer Forschung evaluiert werden, sondern aufgrund des Impact Factors der Zeitschriften, in denen sie publiziert haben [3]. «Qualitäts-Monitoring: yes we count!» lautet der stolze Titel eines kürzlich in der SÄZ erschienen Editorials von Dr. Daniel Herren [4]. In Zukunft zählt das, was gezählt werden kann. Egal ob dies auf falschen Indikatoren beruht - essentiell ist, im Herzen der Ärzteschaft eine neue Art zu installieren, die berufliche Praxis zu begreifen: einen Haufen standardisierter Steine so kostengünstig wie möglich zu produzieren, um auf der Siegerliste der Qualität gut dazustehen. Doch wessen Qualität genau? Eines Haufens von Steinen, die noch nie ein Haus ergeben haben.

\section{Literatur}

1 Projet Monitorage de la qualité de la FMH. www.fmh.ch/fr/themes/qualite/q_monitoring.html

2 Empfehlungen der Schweizerischen Akademie der Medizinischen Wissenschaften. Erhebung, Analyse und Veröffentlichung von Daten über die medizinische Behandlungsqualität. Schweiz Ärztezeitung. 2009;90 (26/27):1044-54.

3 Gori R, Del Volgo MJ. L'idéologie de l'évaluation: un nouveau dispositif de servitude volontaire? Nouvelle Revue de psychosociologie. Janvier 2010.

4 Herren D. Qualitäts-Monitoring: yes we count! Schweiz Ärztezeitung. 2010;91(4):107.

ten. Mit Transparenz über das, was wir bereits tun, mehr Anerkennung zu erreichen, scheint uns ein guter erster Schritt in die richtige Richtung. Frau Treu weist zu Recht auf die verschiedenen Kompetenzen hin, über die ein Spezialarzt für Psychiatrie und Psychotherapie bereits jetzt verfügen muss. Aber wer ausser uns weiss davon? Und wer sonst als die Fachgesellschaften kann definieren, was Qualität in ihrem Fachgebiet bedeutet!

Dr. med. Hans Kurt, Präsident FMPP***

Dr. med. Yvette Attinger Andreoli, Vorstand SGPP, Ressort Qualität Dr. med. Hélène Beutler, Vorstand SGKJPP, Ressort Qualität psychiatri therapie

*** Foederatio Medicorum Psychiatricorum et Psychotherapeuticorum

für Psychiatrie

Schweizerische Gesellschaft für Kinder- und Jugend- 